\title{
El aprendizaje del profesor de matemáticas como campo investigativo $^{1}$
}

\author{
The math teacher's learning as a research field
}

A aprendizagem do professor de matemática como campo de pesquisa

\section{Lida Esperanza Riscanevo Espitia ${ }^{2}$}

Universidad Pedagógica y Tecnológica de Colombia (Colombia)

\author{
Alfonso Jiménez Espinosa ${ }^{3}$ \\ Universidad Pedagógica y Tecnológica de Colombia (Colombia)
}

Recepción: 12/02/2015

Evaluación: 17/05/2015

Aceptación: 05/05/2017

Articulo de Revisión

DOI: https://doi.org/10.19053/01227238.6247

\section{RESUMEN}

Este artículo de revisión presenta diversas paradigmas o marcos conceptuales de formas de entender el aprendizaje del profesor de matemáticas, a través del análisis sobre el foco investigativo relativo al profesor. Profundiza sobre el significado atribuido al aprendizaje del profesor desde los enfoques filosóficos; las problemáticas derivadas de las perspectivas propias del campo; los formación de profesores y las teorías generales de aprendizaje, con el objetivo de comprender los supuestos y conjeturas planteadas, las cuales determinan visiones de aprendizaje del profesor de matemáticas a través de su desarrollo investigativo. Finalmente, considera la perspectiva sociocultural como campo

1 Este artículo hace parte de la revisión teórica y la reflexión sobre el estado del arte del Proyecto de tesis de doctorado de la primera autora, denominado "Arquitecturas de Aprendizaje del Profesor de Matemáticas en diálogo con una experiencia de aprendizaje", del Doctorado en Ciencias de la Educación de la UPTC y dirigida por el segundo autor. Esta tesis enmarca dentro de un proyecto de investigación de mayor alcance, financiado por COLCIENCIAS, intitulado "La problematización de la práctica pedagógica en matemáticas en contextos de investigación colaborativa".

2 Magister en Educación, Estudiante del Doctorado en Ciencias de la Educación RUDE-COLOMBIA, Profesora Asistente del Programa de Licenciatura en Matemáticas, Universidad Pedagógica y Tecnológica de Colombia (UPTC), Integrante del Grupo de Investigación Pirámide. Email: lida.riscanevo@uptc.edu.co

3 PhD en Educación, Profesor Titular del Programa de Licenciatura en Matemáticas de la Universidad Pedagógica y Tecnológica de Colombia (UPTC), director del grupo de investigación Pirámide.Email: alfonso.jimenez@uptc.edu.co 
emergente de desarrollo investigativo, en donde el conocimiento del profesor y el proceso del conocer atribuyen nuevos significados al aprendizaje del profesor de matemáticas.

Palabras clave: Aprendizaje, profesor de matemáticas, teorías de aprendizaje, formación de profesores.

\section{ABSTRACT}

This review article presents several ways to understand the math teacher's learning through the analysis of the research focus regarding the teacher. The meaning conferred to the teacher's learning is deepened from philosophical approaches; the problems derived from the perspectives on the field; the paradigms or conceptual frameworks of teacher training and general theories of learning. The objective consists of understanding the assumptions and conjectures that determine the math teacher's learning view through their research development. Finally, it is considered the sociocultural perspective as an emerging field of research development, where the knowledge of the teacher and the process of knowing attribute new meanings to the math teacher's learning.

Keywords: Learning, math teacher, learning theories, teacher training.

\section{RESUMO}

Este artigo de revisão apresenta diversas formas de entender a aprendizagem do professor de matemática por meio da análise sobre o foco investigativo relativo ao professor. Aprofunda sobre o significado atribuído à aprendizagem do professor a partir dos enfoques filosóficos; as problemáticas derivadas das perspectivas próprias do campo; os paradigmas ou marcos conceituais de formação de professores e as teorias gerais de aprendizagem, com o objetivo de compreender as hipóteses e conjecturas planejadas, as quais determinam visões de aprendizagem do professor de matemática por meio de seu desenvolvimento investigativo. Finalmente, considera a perspectiva sociocultural como um campo emergente de desenvolvimento investigativo, na qual o conhecimento do professor e o processo de conhecer atribuem novos significados à aprendizagem do professor de matemática.

Palavras-chave: Revista História da Educação Latino-americana, Aprendizagem, professor de matemática, teorias de aprendizagem, formação de professores.

\section{INTRODUCCIÓN}

En Educación Matemática se ha interpretado de diversas formas el aprendizaje del profesor de matemáticas. Los marcos referenciales sobre el aprendizaje de los profesores parten del reconocimiento de la gran influencia de los profesores sobre el aprendizaje de los estudiantes. Sin embargo, es necesario problematizarlo a la luz de contextualizar al profesor cuando lleva a cabo actividades de enseñanza 
y no optar simplemente por extender los marcos teóricos del aprendizaje de las matemáticas en los niños ${ }^{4}$.

El foco investigativo relativo al profesor señala que las implicaciones de las investigaciones del aprendizaje del profesor de matemáticas difieren no solo en las hipótesis fundamentales en relación a la naturaleza y propósito de la investigación -aislada versus conectada-, sino también en su concepción de las matemáticas como disciplina - recibida versus construida- y de la enseñanza -libre versus cargada de valores- ${ }^{5}$. Adicionalmente han caracterizado el conocimiento del profesor -conceptualización, dominios y estructura- y la concepción del aprendizaje del profesor -conceptualización y caracterización- ${ }^{6}$.

En este sentido, las investigaciones en la formación de profesores de matemáticas han centrado su mayor interés en la importancia sobre la determinación de los diferentes componentes necesarios del "conocimiento del profesor". Esa intención está ligada a la forma de entender los procesos de construcción de ese conocimiento para enseñar matemáticas; inicialmente, estableciendo relaciones entre el conocimiento teórico y el conocimiento práctico ${ }^{7}$. También han cuestionado el estatus epistemológico de ese constructo, lo cual ha llevado a la adopción reciente del término "conocimiento profesional" el cual se ha usado con diferentes connotaciones; su uso ha llevado a considerar análisis dirigidos a establecer relaciones entre conocimiento y creencias ${ }^{8}$; relaciones entre el conocimiento científico y el conocimiento del profesor, generado a través de la práctica ${ }^{9}$

También se han extendido a establecer relaciones entre el conocimiento, las creencias y la enseñanza y análisis derivados de la integración del conocimiento del profesor y el conocimiento del contenido pedagógico ${ }^{10}$ y relaciones entre conocimientos teóricos y la práctica reflexiva en la constitución de conocimiento práctico del profesor ${ }^{11}$. Estas relaciones también apuntan a reconocer la

4 Stephen Lerman, "A rewiew of research perspectives on mathematics teacher education", en Making sense of mathematics teacher education (Dordrecht, The Netherlands: Kluwer Academic Publishers, 2001), 33-52.

5 Victoria Sánchez García, "La formación de los profesores y las matemáticas. Algunas implicaciones prácticas de las investigaciones", Revista de Educación, No. 306 (1995): 397-426.

6 María Mercedes García Blanco, "La Formación deProfesores de Matemáticas: un campo de estudio y preocupación", Educación Matemática 17, No. 2 (2005): 153-166.

7 Salvador Llinares, "La investigación <sobre> el profesor de matemáticas: aprendizaje del profesor y práctica profesional", Aula 10, (1998):153-179.

8 Gary Fenstermacher, “The Knower and the Know: The Nature of Knowledge in Research in Education", en Review of Research in Education, eds. L. Darling-Hammond (Washington: AREA, 1994).

Thomas Cooney y T. Wilson, "Teachers Thinking About Functions: Historical and Research Perspectives", en Integrating Research on the Graphical Representation of Functions, eds. T. Romberg, E. Fennema, y T. Carpenter (Hillsdale, NJ: Lawrence Erlbaum Associates, 1994)

9 Donald Schön, The reflective practitioner (New York: Basic Books, 1983)

Donald Schön, Educating the reflective practitioner.Toward a new desig for teaching and learning in the professions (San Francisco: Jossey- Bass, 1987).

10 Lee Shulman, "Those who understand: knowledge growth in teaching" Educational Researcher, (1986): 4-14. Lee Shulman, "Renewing the pedagogy of teacher education: The impact of subject-specific conceptions of teaching", en Las didácticas especificas en la formación de profesores, eds. L. Moreno y J.M. Vez (Santiago: Tórculo, 1993): 53-69.

Lee Shulman, "Foreword", en Examining Pedagogical Content Knowledge, eds. J. Gess-Newsome y N. Lederman (Londres: Kluwer Academic Publishers, 1999): ix-xii.

11 Llinares, "La investigación <sobre> el profesor de matemáticas: aprendizaje del profesor y práctica profesional" 
singularidad del conocimiento del profesor la cual le permite desarrollarse cada vez más de forma competente $\mathrm{e}^{12}$. Esta singularidad "se manifiesta cuando se intenta explicitar las diferencias en la manera de conocer el contenido matemático por un profesor $y$ por alguien que no es profesor"13 generando implícitamente la necesidad de hacer diferencia entre el conocimiento matemático especializado y el conocimiento matemático común. Siguiendo a Ball, Thames y Phelps ${ }^{14}$ el conocimiento que es usado en contextos de enseñanza para resolver las tareas específicas de enseñar matemáticas debe reconocerse como especializado, el conocimiento que usan o usamos estudiantes, profesores o gente del común en su diario vivir para resolver problemas de matemáticas debe reconocerse como común. En este sentido, se torna relevante la forma como el conocimiento del profesor "pone de relieve la importancia de los contextos de uso del conocimiento y las tareas profesionales que lo definen y, en particular, la manera en la que el conocimiento permite al profesor realizar con competencia una determinada práctica"15.

Estas diferentes formas de asumir el conocimiento profesional del profesor de matemáticas se ven reflejadas en desarrollos investigativos. En un primer momento se presentan sus avances investigativos cuestionando qué conocimiento necesita el profesor; de esta forma se hace énfasis en la naturaleza del conocimiento matemático y en cómo se adquiere ese conocimiento, lo que permite asumir al profesor de matemáticas como agente cognitivo y reflexivo ${ }^{16}$. En un segundo momento se hace énfasis en una concepción del conocimiento matemático como un proceso social y cultural ${ }^{17}$. Las ideas conceptuales que surgen a través de entender el conocimiento matemático bajo esta visión sociocultural y las relaciones que se establecen sobre la práctica profesional, las creencias y las relaciones entre los diferentes dominios de otros conocimientos, resaltan el conocimiento profesional como algo más complejo.

En este sentido, Azcárate ${ }^{18}$ asume el conocimiento profesional como contextual, interactivo, especulativo, situado, no parcelado, de carácter práctico y personal y adaptable a contextos determinados. Es decir, se requiere de una visión integradora y gradual de diversas fuentes de conocimiento que conlleven a la solución de problemas que tengan como finalidad práctica la enseñanza.

12 Salvador Llinares, "Experimentos de enseñanza e investigación. Una dualidad en la práctica del formador de profesores de matemáticas", Educación Matemática, no extraordinario XXV aniversario, (2014): marzo, 31-51.

13 Llinares, "Experimentos de enseñanza e investigación”, 31.

14 Debora Ball, Mark Thames y Geoffrey Phelps, "Content knowledge for teaching: What makes it special?", Journal of Teacher Education 59, No 5 (2008): 389-407.

15 Llinares, "Experimentos de enseñanza e investigación”, 32.

16 Thomas Cooney, "Research and Teacher Education: in search of common ground", en Journal for Research in Mathematics Educations 25, (1994): 608-636.

María Peñas, "Un estudio del proceso de reflexión sobre cuestiones profesionales en la formación inicial de profesores de matemáticas," en Sexto Simposio de la Sociedad Española de Investigación en Educación Matemática, eds. Jesús Murillo, Petra María Arnal, Rafael Escolano, José María Gairín, y Lorenzo Jesús Blanco (SEIEM. Universidad de la Rioja. Departamento de Matemáticas y Computación, 2003).

17 Stephen Lerman, "Socio-cultural research in PME", en Handbook of Research on the Psichology of Mathematics: past, Present and Future (Rotterdam-Tapei: Sence Publishers, 2006): 347-366.

18 Pilar Azcárate, "La formación inicial del profesor de matemáticas: análisis desde la perspectiva del conocimiento práctico profesional", Revista Interuniversitaria de Formación del Profesorado (1998): 129-142 
De esta forma este conocimiento viene a depender de las situaciones en que se adquiere, se aprende y se usa, lo cual da cabida al conocimiento del profesor desde la perspectiva de la cognición situada ${ }^{19}$.

La forma de entender el conocimiento matemático y su relación con la formación de profesores, en los últimos años son determinante en nuevas posibilidades investigativas. Se puede considerar que características asociadas a las perspectivas situadas de la cognición situada, la cognición como social y la cognición como distribuida son relevantes frente a la consideración del conocimiento matemático, desde una perspectiva sociocultural. Sin embargo, no en la misma forma de desarrollo, esta visión sociocultural focaliza el aprendizaje del profesor de matemáticas como campo de investigación.

En consecuencia, estos desarrollos investigativos sobre el aprendizaje del profesor de matemáticas se ponen de relieve en este artículo, dado que estos desarrollos teóricos generan un campo emergente que problematiza posicionamientos sobre la formación docente, la relación teoría y práctica, la conceptualización del aprender a enseñar, la investigación con el profesorado y los conocimientos necesarios para ser profesor de matemáticas.

\section{Desafíos frente al Aprendizaje del Profesor de Matemáticas como Campo Investigativo}

La necesidad de dirigir esa problematización hacia otros focos investigativos motiva el seguimiento de las diferentes problemáticas propias del aprendizaje del profesor de matemáticas, como campo de investigación en la formación de profesores. En este sentido, interrogantes o afirmaciones realizadas por investigadores en los últimos años, se plantean como desafíos, los cuales presentan herramientas conceptuales de gran interés propicias para problematizar el proceso de aprendizaje del profesor.

Se parte de considerar el siguiente interrogante: ¿qué investigación se está produciendo que pueda contribuir a la enorme necesidad de apoyar el aprendizaje y desarrollo de los profesores? ${ }^{20}$ En él se proyecta la necesidad de comprender cómo aprenden los maestros, con qué oportunidades, bajo qué condiciones y cómo mejorar las oportunidades de los profesores para aprender. Esta necesidad es considerada un reto, al igual que "determinar la importancia del carácter específico del dominio de las matemáticas ${ }^{\prime 21}$, en donde asumir una teoría de aprendizaje de los

19 Maria García, "La formación inicial de profesores de matemáticas. Fundamentos para la definición de un currículum”, en A formação de professores de matemática. Estudos e contribuições teorico-metodologicas de Brasil Espanha e Portugal, eds. Dario Fiorentini (Campinas, Brasil : UNICAMP, 2001).

20 Jill Adler, Deborah Ball, Konrad Krainer, Fou-Lain Lin, y Jarmila Novotna, "Mirror images of an emerging field: researching teacher education" en Proceedings from the ICME 10 (Dinamarca: IMFUFA, Departament of Sicence, Systems and Models, Roskilde University Denmark, 2008):123-139.

http://static-content.springer.com/lookinside/art\%3A10.1007\%2Fs10649-005-5072-6/000.png (18 de junio, 2014) o DOI: 10.1007/ s19649-005-5072-6

21 Konrad Krainer, "Individuals, teams communities and networks: participants and ways of participation in mathematics teacher education," en Participants in Mathematics Teacher Education, eds. Konrad Krainer y Terry Wood (Rotterdam: Sence Publishers, 2008), 10. 
profesores es un tema clave para la investigación sobre la formación del profesorado de matemáticas ${ }^{22}$ y sobre el cual se señala como una problemática propia de este abordar la relación de la práctica de los formadores con el aprendizaje de profesores y de estudiantes ${ }^{23}$.

En este sentido, algunas investigaciones ${ }^{24}$ subrayan la necesidad de fundamentar los programas de formación de profesores en un conjunto explícito de teorías del aprendizaje del profesor, planteando la necesidad de incorporar en los programas de formación los diferentes elementos de conocimiento necesarios para desarrollar la práctica de enseñar matemáticas; es decir, al diseño de oportunidades de aprendizaje en las que los estudiantes para profesor puedan tener la posibilidad de dotar de significado y usar los medios que les permitan desarrollar ese conocimiento ${ }^{25}$. La consideración de las oportunidades de aprendizaje resalta la vinculación de los profesores a comunidades, grupos o redes ${ }^{26}$, en donde las investigaciones del aprendizaje que favorecen estas oportunidades deben considerar "que el aprendizaje de los maestros es un proceso complejo y es en gran medida influenciado por factores personales, sociales, organizacionales, culturales y políticos" 27 .

De otro lado, esta consideración también exalta la relación entre la formación inicial y el desarrollo profesional de los profesores. Esta relación se apoya en "caracterizar el proceso de aprendizaje de los estudiantes para profesor y los procesos de desarrollo profesional de los profesores en ejercicio desde el mismo modelo de aprendizaje"28. Se enfatiza en los diseños como entornos para el aprendizaje, sobre los cuales se determinan variadas formas de significar la intrínseca relación entre cognición situada y las comunidades de práctica o también llamadas en este caso comunidades de aprendizaje; asumiendo el aprendizaje como un proceso a lo largo de la vida y un proceso que se vive colectivamente ${ }^{29}$. La relación entre la cognición situada y las comunidades de práctica, se justifica en algunos casos por asumir el carácter integrado del conocimiento del profesor, por estar conectado con la práctica; es

22 Este interrogante surge en uno de los más recientes meta estudios sobre formación de profesores de matemáticas organizado para la Comisión Internacional de Instrucción Matemática (ICME-10) Este metaestudio se realizó a partir de 160 trabajos de investigación en formación de profesores, todos ellos publicados en revistas reconocidas en el campo de la Educación Matemática.

23 Jill Adler y Bárbara Jaworski, “The state of research on mathematics teacher education, and how it needs to develop," Trabajo presentado en The Fifteenth ICMI Study (Aguas de Lindóia, 2005).

24 Lorenzo Blanco, "Problem solving and the inicial and theoretical education of teachers in Spain," en Mathematics Teacher Educational and Development 6, (2004):37-48.

José Carrillo Yáñez y Nuria de los Ángeles Climent Rodríguez, Modelos de formación de maestros en matemáticas, (Huelva: Servicio de Publicaciones de la Universidad de Huelva,1999).

C.Corral y E. Zurbano, Propuestas metodológicas y de evaluación en la formación inicial de los profesores del área de didáctica de la matemática (Oviedo: Servicio de Publicaciones Universidad de Oviedo, 2000).

Joaquín Giménez, Salvador Llinares y Victoria Sánchez, El proceso de llegar a ser un profesor de matemáticas. Cuestiones desde la Educación Matemática (Granada: Comares, 1996).

25 Salvador Llinares "Aprendizaje del estudiante para profesor de matemáticas y el papel de los nuevos instrumentos de comunicación," en conferencia realizada en el marco del III Encuentro de Programas de Formación Inicial de Profesores, Universidad Pedagógica Nacional en Colombia, 2008. http://rua.ua.es/dspace/bitstream/10045/5302/1/linares-bogota08.pdf

26 Stephen Lerman y Stefan Zehetmeier, "Face-to-Face Communities and Networks of Practicing Mathematics Teachers Studies on their professional growth,"en Handbook Communities and Networks of Mathematics Teachers as Learners 3, eds. Konrad Krainer y Terry Wood (Rotterdam: Sence Publishers, 2008):133-154.

27 Konrad Krainer, "Individuals, teams communities and networks", 10.

28 Salvador Llinares, "Formación de Profesores de Matemáticas. Desarrollando Entornos de Aprendizaje àra relacionar la formación inicial y el desarrollo profesional. Conferencia invitada en el XIII Jornadas de Aprendizaje y Enseñanza de las Matemáticas. (Granada, España, Julio de 2007).

29 Y. Chávez y Salvador Llinares, "La identidad como producto del aprendizaje en la práctica de enseñar matemáticas en profesores de primaria”, en Investigación en Educación Matemática XVI, eds. A. Estepa, A. Contrreras, J. Deulofeu, M. Penalva y L. Ordóñez (Jaén: SEIEM,2012):187-196. 
decir, el conocimiento se genera a través de la experiencia práctica vinculada a problemas concretos; es así, como el proceso de aprender a enseñar matemáticas puede ser concebido como un proceso de enculturación ${ }^{30}$ en el cual la naturaleza del conocimiento y las creencias se construyen, hecho que permite abordar el aprendizaje como proceso social.

El seguimiento de estos cuestionamientos y afirmaciones recientes sobre el aprendizaje del profesor de matemáticas permite reconocer que este campo investigativo se ha alterado a lo largo del tiempo a través del significado atribuido a aprender a enseñar, sobre la base de interpretar la naturaleza del contenido matemático, la organización del conocimiento del profesor, el uso de ese conocimiento en situaciones de enseñanza ${ }^{31}$ y la relación que se establece entre el conocimiento teórico y el conocimiento generado en la práctica ${ }^{32}$.

\section{Visiones sobre el Aprendizaje del Profesor de Matemáticas}

La problematización sobre el aprendizaje del profesor prioriza establecer un análisis sobre el significado atribuido al aprendizaje del profesor de matemáticas en los paradigmas o marcos conceptuales de formación de profesores, teorías generales de aprendizaje, enfoques filosóficos y líneas de investigación propias del campo. Este análisis resalta la necesidad de asumirlo como foco investigativo emergente desde las perspectivas socioculturales, a través de la identificación de visiones de aprendizaje del profesor de matemáticas en los desarrollos investigativos.

La primera visión se encuentra bajo el enfoque filosófico desde la perspectiva científico-analítica de Brown \& Borko ${ }^{33}$. Esta perspectiva pone de relieve la naturaleza, libre de valores de la ciencia, manteniendo cierta distancia entre el investigador y el problema objeto del estudio. Bajo esta perspectiva se encuentran investigaciones enmarcadas en el paradigma tradicional-artesano de formación de profesores ${ }^{34}$, que concibe la enseñanza como un cuerpo de conocimientos del oficio; el marco conceptual denominado práctico-oficio de Feiman-Nemser ${ }^{35}$ el cual privilegia la experiencia como fuente de conocimiento sobre la enseñanza y sobre el aprender a enseñar; y el modelo práctico-aislado ${ }^{36}$, donde solo los

30 Salvador Llinares, "Relación entre teorias sobre el aprendizaje del profesor de matemáticas y diseños de entornos de aprendizaje," en Conferencia invitada presentada en el Congreso Iberoamericano de Educación Matemática- CIBEM (Portugal, 2005).

31 Llinares, "La investigación <sobre> el profesor de matemáticas" Lerman "A rewiew of research perspectives on mathematics teacher education" Gómez, "Teorías de aprendizaje y formación de profesores"

32 María Mercedes García y Victoria Sánchez, "Las perspectivas socioculturales y la formación de profesores de primaria en relación con las matemáticas"

33 Catherine Brown y Hilda Borko, "Becoming a Mathematics Teachers", en Handbook of Research on Mathematics Teaching and Learning, eds. A. Grouws (New York: MacMillan, 1992): 209-239.

34 Kenneth Zeichner, "Connecting genuine teacher development to the struggle for social justice" en Journal of Education for Teaching 19, no 1 (1993): 5-20.

35 Sharon Feiman-Nemser, “Teachers preparation:structural and conceptual alternatives" en Handbook of research on teacher education, eds. W. Houston (New York: MacMillan,1990).

36 Dario Fiorentini, "De professor isolado ou plugado para professor conectado: novas perspectivas à formação do professor de matemáticas" en Coletânea de trabalhos do PRAPEM-VII ENEM (Campinas: CEMPEM/PRAPEM-FE/UNICAMP, 2001). 
conocimientos en matemáticas serían suficientes para ser buen profesor, donde además, aspectos básicos como "por qué", "para qué" y "cómo enseñar" se aprenden en la práctica.

De esta perspectiva se derivan tres tendencias. La primera tendencia se identifica por asumir que "el arte de enseñar se aprende enseñando"; esto es, en la práctica; no habiendo necesidad de una formación específica o teórica sobre las relaciones entre la matemática, el alumno y el profesor. En ese contexto, el aprendizaje docente se presenta de forma artesanal ${ }^{37}$; es decir, el aprendizaje del oficio basado en la práctica que se realiza a través de ensayo y error. La experiencia como estudiante de matemáticas en la escuela, universidad o instituto es determinante, pues alude al aprendizaje por observación, por vía oral o por imitación. En esta tendencia se mira el aprendizaje como la modificación de la conducta por la experiencia ${ }^{38}$.

La segunda tendencia se fundamenta en el profesor eficaz, la cual encaja dentro de la llamada tradición de la psicología cognitiva de Brown \& Borko ${ }^{39}$, inscrita en el paradigma proceso-producto. Esta se relaciona con el marco conceptual de formación de profesores, denominado tecnológico por FeimanNemser $^{40}$ y el modelo técnico-colgado ${ }^{41}$, en donde el profesor primero aprende la teoría para después aplicarla en la práctica. Aquí la práctica se ve como un campo de entrenamiento y de experimentación. Esta tendencia se identifica con investigaciones que procuran relacionar el aprendizaje del profesor con comportamientos, metodologías, disposición física de las clases y resultados de aprendizaje de los estudiantes medidos a través de test estandarizados.

En esta última tendencia se ve la práctica de enseñanza como campo de aplicación de conocimientos producidos sistemáticamente por la investigación académica $^{42}$, donde el problema de la enseñanza es un problema de desarrollar en los profesores la habilidad para llevar a la práctica un conjunto de procedimientos específicos que se consideran eficientes -desde la perspectiva del rendimiento de sus alumnos- ${ }^{43}$. Esta caracterización fue privilegiada en las décadas de los setenta y ochenta, periodo que puede entenderse como un primer momento en el cual la formación de profesores de matemáticas no solo se contempla como área de actuación, sino de investigación ${ }^{44}$, que privilegia una perspectiva investigativa cuantitativa de corte positivista ${ }^{45}$.

\footnotetext{
37 Dario Fiorentini y Ana Teresa de Carvalho Correa de Oliveira, "Lugar das matemáticas na Licenciatura em Matemáticas: que matemáticas e que práticas formativas", Boletim de Educação Matemática 27, no 47 (2013): 917-938.

38 Gómez, "Teorías de aprendizaje y formación de profesores,"

39 Brown y Borko, "Becoming a Mathematics Teachers"

40 Feiman-Nemser, “Teachers preparation: structural and conceptual alternatives"

41 Fiorentini, "De professor isolado ou plugado para professor conectado"

42 Fiorentini y Carvalho, "Lugar das matemáticas na Licenciatura em Matemáticas"

43 Gómez, "Teorías de aprendizaje y formación de profesores,"

44 Fou-Lain Lin y Thomas Cooney, Making sence of mathematics teacher education (Dordrecht The Netherlands: Kluwer, 2001) citado por Cristina Esteley, "Desarrollo Profesional en Escenarios de Modelación Matemática: voces y sentidos" (Tesis de Doctorado en Universidad Nacional de Córdoba, 2010).

45 Stephen Brown, Thomas Cooney, Douglas Jones, "Mathematics Teacher Education," en Handbook of Research on Teachers Education, editado por W. Houston ( New York: MacMillan, 1990).
} 
La tercera tendencia derivada desde la línea de investigación de la psicología cognitiva, establece que la consideración epistemológica del constructivismo en la formación de profesores de matemáticas, no es ajena a la entendida por la concepción constructivista del aprendizaje matemático, según la cual se construye conocimiento a partir de las estructuras cognitivas ${ }^{46}$. En esta tendencia se consideran las creencias y concepciones de las matemáticas y su enseñanza en los profesores y futuros profesores, como estructuras cognitivas anteriores; además establece las formas en que las estructuras cognitivas se conforman, cambian y se movilizan en la práctica ${ }^{47}$.

Estas características parten de suponer que la formación es un proceso individual, en el cual el profesor modifica y desarrolla sus estructuras cognitivas partiendo de perturbaciones generadas por su propia experiencia. El profesor "construye su conocimiento y lo adapta a esa experiencia en actividades que significan un reto, ponen en juego su conocimiento para generar conflictos $\operatorname{cognitivos}^{\prime 48}$. Se identifica en esta tendencia el paradigma de la formación orientada a la indagación de Zeichner ${ }^{49}$, la cual se centra en la forma como el profesor interpreta su proceso formativo a partir del reconocimiento de la naturaleza problémica de la enseñanza, pues la concibe como un conjunto de orientaciones y destrezas de carácter reflexivo y crítico.

Esta tendencia se interpreta en dos sentidos, los que estudian los significados atribuidos por los profesores a las matemáticas, a la enseñanza y al aprendizaje de los estudiantes y los que, a partir de esos significados están interesados en determinar las influencias de condiciones sociales ${ }^{50}$; es decir, los procesos de socialización del profesor. El primer sentido ubica una de las dos perspectivas de las investigaciones en formación de profesores de matemáticas, que según Lerman $^{51}$, ha caracterizado las investigaciones en los años noventa, centradas en el profesor y sus creencias que presentan una visión individualista e internalista del aprendizaje. Unas buscan categorizar y clasificar las creencias del profesor, y otras buscan controlar los cambios en dichas creencias, pero además establecen una relación causa-efecto entre las creencias y la práctica, sin considerar relevante el contexto de producción tanto de las creencias como de las prácticas.

Sobre esta visión del aprendizaje desde la perspectiva científico-analítica se puede inferir que su desarrollo investigativo se presenta en el marco teórico

46 Pablo Flores, "Concepciones y creencias de los futuros profesores sobre las matemáticas, su enseñanza y aprendizaje" (Tesis de Doctorado en Universidad de Granada, 1998) citando a Ernest Von Glasersfeld,"Cognition, construction of knowledge, and teaching," Synthese. Vol.:80 (1989):121-140.

Gérard Vergnaud, "Epistemology and psychology of mathematics education," en Research Sinthesis by International Group for the Psychology of Mathematics Education, eds. J. Pearla Nesher y Jeremy. Kilpatrick ( New York: Cambridge University Press, 1990): 14-30.

Paul, Ernest, "What is social constructivism in the psychology of mathematics education?" en Prodeedings of the Eighteenyh International Conference for PME, eds. Joao Ponte, y Joao Filipe Matos (Lisboa, 1994): 304-311.

Stephen Lerman, "Metaphors for mind aand mataphors for teaching and learning mathematics," en Proceeding of the Eigghteenth International Conference for PME, III, J eds. Joao Ponte, y Joao Filipe Matos (Lisboa, 1994):144-151.

47 Peñas, Un estudio del proceso de reflexión sobre cuestiones profesionales en la formación inicial de profesores de matemáticas,-

48 Gómez, "Teorías de aprendizaje y formación de profesores" 7.

49 Zeichner, "Connecting genuine teacher development to the struggle for social justice"

50 Brown, Cooney y Jones, "Mathematics Teacher Education"

51 Lerman, "A rewiew of research perspectives on mathematics teacher education". 
de la psicología cognitiva, partiendo de asumir que en la mente humana el conocimiento se organiza y almacena en estructuras y que además estas estructuras de conocimiento y las representaciones mentales que generan son relevantes en las percepciones individuales ${ }^{52}$, lo cual genera que

[...] aprender a enseñar supone la adquisición de sistemas de conocimiento («schemata»), destrezas cognitivas tales como resolución de problemas y toma de decisiones pedagógicas, y un conjunto de comportamientos de enseñanza observables. Para comprender el proceso de aprender a enseñar, uno debe estudiar cómo estos sistemas - y las relaciones entre ellos - se desarrollan y cambian con la experiencia, así como identificar los factores que influencian este proceso de cambio ${ }^{53}$.

La segunda visión del aprendizaje se enmarca desde la perspectiva humanista de Brown \& Borko ${ }^{54}$, en la cual se identifica el marco conceptual crítico social de la formación de profesores de Feiman-Nemser ${ }^{55}$, y el paradigma orientado a la indagación de Zeichner ${ }^{56}$, sobre los cuales se pueden identificar dos tendencias investigativas.

La primera tendencia derivada del proceso de socialización del profesor tiene como objetivo comprender el proceso por el que un individuo llega a ser un profesor; en este proceso se puede distinguir el enfoque funcionalista, el interpretativo y el crítico ${ }^{57}$. El funcionalista se enfoca principalmente a describir los factores externos que influyen sobre los profesores; el interpretativo destaca la gran cantidad de influencias que condicionan las elecciones de los profesores, centrándose en las experiencias individuales en contraposición a las tendencias colectivas; y el crítico, trata de clarificar el papel que juegan las desigualdades en el contexto de socialización del profesor ${ }^{58}$.

En esta tendencia investigativa se privilegia el constructivismo social, el cual argumenta que el conocimiento necesario para enseñar se genera a partir de las actividades que se realizan en diversos tipos de tareas. Supone que el poder del intelecto depende de herramientas social y culturalmente constituidas para apropiar la cultura y la historia, dando importancia a la interacción del profesor y sus compañeros como pares que apoyan la construcción del conocimiento y de las herramientas que sustentan la mediación entre la mente y la experiencia ${ }^{59}$.

\footnotetext{
52 Sánchez, "La formación de los profesores y las matemáticas" Llinares, "La investigación <sobre> el profesor de matemáticas: aprendizaje del profesor y práctica profesional”

53 Catherine Brown y Hilda Borko, “Becoming a Mathematics Teachers," en Handbook of Research on Mathematics Teaching and Learning, eds. A. Grouws (New York: MacMillan,1992) :212.

54 Brown y Borko, "Becoming a Mathematics Teachers"

55 Feiman-Nemser, “Teachers preparation: structural and conceptual alternatives"

56 Zeichner, "Connecting genuine teacher development to the struggle for social justice"

57 K. Zeichner y J. Gore, "Teacher Socialization", Paper presented at the annual meeting of the Association of Teacher Educators (Las Vegas, February de 1990).

58 Zeichner y Gore, "Teacher Socialization”,

59 Gómez, “Teorías de aprendizaje y formación de profesores,"
} 
La segunda tendencia se centra en los profesores y sus $\operatorname{contextos}^{60}$, la cual privilegia el conocimiento profesional del profesor de matemáticas. Estas investigaciones centradas en el desarrollo profesional conciben al profesor como un adulto aprendiendo el proceso de llegar a ser profesor, en donde su desarrollo es el resultado de cambios evolutivos en sus estructuras cognitivas ${ }^{61}$. Se asume que el conocimiento debe ser integrado, sin limitar su aprendizaje; es decir, miran el aprendizaje -incluido el aprendizaje sobre la enseñanza- como actividad sociocultural, lo cual implica que el aula es un lugar complejo, de influencias políticas y sociales. El análisis de las interacciones socioculturales que allí emergen plantean posiciones múltiples y divergentes sobre clases, género, minorías, relaciones profesor-estudiante y otras prácticas discursivas donde las relaciones de poder emergen naturalmente ${ }^{62}$.

Esta segunda tendencia investigativa se interpreta en cuatro sentidos. El primero, desde la perspectiva psicológica entiende al individuo como sujeto social ${ }^{63}$ que considera al profesor como un aprendiz, en términos de Oliveira \& Hannula ${ }^{64}$, y Perrin-Glorian, Deblois \& Robert ${ }^{65}$. Tres puntos de vista sobre el aprendizaje del profesor de matemáticas que lo asumen como individuo, han sido abordados por las diferentes investigaciones hasta el momento. Aquellos que perciben el aprendizaje como la adquisición de conocimientos o creencias; como el dominio de una habilidad, por ejemplo la capacidad para observar y reflexionar, que en algunos casos asume la práctica del profesor en el aula de clase como situaciones didácticas que le permiten vivir y analizar como los estudiantes establecen su relación con el conocimiento ${ }^{66}$; y cómo la adopción de una cierta disposición, por ejemplo, una identidad o una orientación ${ }^{67}$. Este concepto de identidad, también se presenta a través de analizar el proceso de convertirse en maestros, indagando formas de asumir el desarrollo profesional e interpretar la cultura escolar, para identificar los factores que se considera inciden en la capacidad de convertirse en profesionales eficaces como lo señala Prescott y Cavanagh ${ }^{68}$.

El segundo sentido, desde las teorías socioculturales, considera el aprendizaje reflexivo, que asume que "la interacción y el contraste con los demás, con uno

60 Lerman, "A rewiew of research perspectives on mathematics teacher education".

61 Gómez, "Teorías de aprendizaje y formación de profesores,"

62 Lerman, "A rewiew of research perspectives on mathematics teacher education".

63 Krainer, "Individuals, teams communities and networks: participants and ways of participation in mathematics teacher education"

64 Hélia Oliveira y Markku Hannula, "Individual prospective mathematics teachers," en The International Handbook of Mathematics Teachers Education 3, eds. Konrad Krainer y Terry Wood (Rotterdam: Sence Publishers, 2008): 13-34.

65 Marie-Jeanne Perrin-Glorian, Lucie DeBlois y Aline Robert, "Participants in Mathematics Teacher Education: Individual, Teams, Communities and Networks," en The International Handbook of Mathematics Teacher Education 3, eds. Konrad Krainer y Terry Wood (Rotterdam: Sence Publishers, 2008): 35-59.

66 C. Margolinas, C. y M. J. Perrin-Glorian, "Des recherches visant à modéliser le rôle de l'enseignant", Recherches en didactique des mathématiques 17, no 3 (1997): 7-15.

M. Hersant y Marie-Jeanne Perrin-Glorian. "Characterization of an Ordinary Teaching Practice with the Help of the Theory of Didactic Situations", Educational Studies in Mathematics, 59, no 1-3 (2005): 113-151.

67 Oliveira y Hannula, "Individual prospective mathematics teachers"

68 Prescott y M. Cavanagh, "A situated Perspective on Learning to Teach Sencondary Mathematics", En Proceedings of the 31st Annual Conference of the Mathematics Education Research Group of Australian, eds. R. Goos, R. Brown y K. Makar (Merga Inc,2008). 
mismo y la teoría posibilitan la co-construcción y la reconstrucción activa de conocimientos $^{\prime 69}$. Este planteamiento se complementa con el aprendizaje realista como modelo de formación de profesores de matemáticas; el cual se fundamenta en la Educación Matemática realista, la perspectiva sociocultural de aprendizaje humano de Vigostky ${ }^{70}$ y el aprendizaje reflexivo de Schön ${ }^{71}$.

El tercer sentido destaca la importancia del contexto como determinante del significado de los conceptos, pues los considera como herramientas que permiten participar en las prácticas de enseñar matemáticas ${ }^{72}$. El aprendizaje del profesor se ve como un proceso de enculturación en una comunidad de prácticas $^{73}$, analizando cómo los estudiantes para profesor se apropian de instrumentos para pensar y actuar en comunidades de práctica. Enfatiza en el conocimiento visto como el uso de los instrumentos, y el aprender como la transformación de la persona mediante la participación en las prácticas sociales. Aprender a enseñar matemáticas consiste en la generación y uso de una serie de instrumentos técnicos y conceptuales y, en las diversas tareas profesionales vinculadas a la enseñanza de la matemática.

Se establece una relación con las teorías del aprendizaje situado, en donde el conocimiento es situado, la cognición es distribuida y los conceptos se ven como herramientas que permiten participar en prácticas y cuyo uso depende del contexto $^{74}$. El carácter situado del conocimiento,

[...] subraya la importancia de los contextos y del tipo de actividades en la generación del conocimiento, así como del conocimiento que poseen los profesores y las situaciones en las que se adquiere y usa, se asume que el conocimiento es inseparable de los contextos y las actividades en los que se desarrolla ${ }^{75}$.

El cuarto sentido está orientado desde perspectivas no psicológicas. En este caso, "el aprendizaje es un fenómeno esencialmente social. El aprendizaje como práctica social destaca el significado, la práctica, la identidad y la comunidad como elementos constituyentes del aprendizaje" ${ }^{\prime 76}$. Desde esta perspectiva se privilegia tópicos sobre el aprendizaje del profesor como participación en la práctica; estas teorías ofrecen un modo de interpretar el aprendizaje de los profesores como participación social y en construir identidades en relación con

69 Angel Alsina, "El aprendizaje reflexivo en la formaión inicial del profesorado: un modelo para aprender a enseñar matemáticas" Educación Matemática 22, no 1 (2010):149.

Alfonso Jiménez Espinosa, "Quando professores da Matemática da escola e da universidade se encontram: re-signifição e reciprocidade de saberes (Tese de Doutorado em Educação; Universidade Estadual de Campinas, 2002.

70 Lev Vigostki, Pensamiento y Lenguaje (La Habana: Editorial Revolucionaria, 1978).

71 Schön, The reflective practitioner

72 Gómez, "Teorías de aprendizaje y formación de profesores,"

73 Llinares, "Relación entre teorias sobre el aprendizaje del profesor de matemáticas"

74 Llinares, "Relación entre teorías sobre el aprendizaje del profesor de matemáticas y diseños de entornos de aprendizaje,"

75 García Blanco, "La Formación de Profesores de Matemáticas", 157.

76 Etiene Wenger, Comunidades de Práctica. Aprendizaje, significado e identidad (Barcelona: Ediciones Paidos Ibérica, S. A, 2001). Etiene Wenger, "Uma teoria social da aprendizagem”, en Teorias contemporâneas da aprendizagem, eds. Knud Illeris (Porto Alegre: Penso, 2013). 
esas comunidades sociales ${ }^{77}$. Según Camargo ${ }^{78}$, los trabajos de investigación en formación de profesores desde esta perspectiva son de dos tipos:

[...] en unos se asumen que el entorno usual de formación, o de desempeño profesional, es de hecho una comunidad natural de práctica y los investigadores buscan identificar los efectos de la misma en el aprendizaje (...). En otros, se busca generar espacios de formación de profesores, o de futuros profesores, en donde ellos hagan parte de una comunidad de práctica conformada a propósito alrededor de un asunto problemático; el aprendizaje se documenta dando cuenta de la evolución de la participación o de la identidad profesional.

Estas investigaciones reflejan un primer interés de desarrollo investigativo, enfocado a la reflexión sobre la enseñanza de las matemáticas en conformación de comunidades de práctica de estudiantes en formación inicial y formadores. El punto en común de este interés investigativo, está en considerar el análisis de las prácticas sobre la enseñanza de las matemáticas como eje principal de estudio y la caracterización del análisis de las prácticas como estrategia reflexiva de formación ${ }^{79}$; el desarrollo del concepto de "comunidades de prácticas", a partir de cuestionar, cómo una comunidad aprende y transforma sus prácticas, sobre todo sus discursos y saberes sobre formación de profesores en matemáticas, en un contexto de cambios curriculares ${ }^{80}$.

Un segundointerés de investigación presenta tópicos teóricos y metodológicos que fundamentan proyectos dirigidos a la construcción de comunidades de investigación entre profesores y didácticas, para mejorar el aprendizaje de las matemáticas. Se asumen las conceptualizaciones teóricas sobre investigación y comunidades como relevantes, en el sentido de asumir la investigación como oportunidad de aprendizaje. Abordan los modos de afiliación propuestos por Wenger como características asociadas a la construcción de comunidades, planteando la necesidad de abordar la identidad tanto individual como colectiva $^{81}$, a través de proponer un análisis de las interacciones dialógicas dentro de la comunidad, como una forma de significar su aprendizaje. La participación y utilización de artefactos en la conformación y desarrollo de las comunidades de práctica permiten la negociación de significado al establecer relaciones entre sí, desarrollar su identidad y buscar ser miembros de esa comunidad. Se

77 Chávez y Llinares, "La identidad como producto del aprendizaje en la práctica de enseñar matemáticas en profesores de primaria"

78 Leonor Camargo, "Descripción y análisis de un caso de enseñanza y aprendizaje de la demostración en una comunidad de práctica de futuros profesores de matemáticas de educación secundaria” (Tesis de Doctorado en Universidad de Valencia, Valencia, España: 2010).

79 Pedro Gómez, "Procesos de Aprendizaje en la Formación Inicial de Profesores de Matemáticas de Secundaria", Electronic Journal of research in Educational Psychology 7, No. 1 (2009): 471-498.

80 J. Melo, “ A formação do formador de professores de matemática no contexto das mudanças curriculares (Tesis de doctorado en, Campinas, São Paulo, Brasil: Universidade Estadual de Campinas, 2010)

81 Bárbara Jaworski, "Building and sustaining inquiry communities in mathematics teaching development. Teachers and didacticians in collaboration", en Participants in Mathematics Teachers Education, eds. K. Krainer, y T. Wood (Sence Publishers. All rights reserved, 2008):309-33 
connota una nueva interpretación de aprender a enseñar como un "proceso de convertirse" ${ }^{\prime 2}$.

Finalmente, un tercer interés investigativo recae en una línea de trabajo que vincula el aprendizaje con el desarrollo profesional ${ }^{83}$. Resaltan los procesos de participación y cosificación de los participantes en comunidades investigativas, a través de analizar las practicas investigativas, derivadas de problematizar el proceso de enseñar y aprender matemáticas en contextos colaborativos de investigación. En estas investigaciones, el concepto de comunidad de prácticas se asume, como un modo de investigar el aprendizaje en comunidades investigativas, pues su desarrollo a lo largo del tiempo constituye su identidad.

Este sentido considera al profesor como reflexivo e investigativo y se identifica con el modelo del profesor conectado ${ }^{84}$, en donde el profesor es alguien que participa del debate público, participa colectivamente de proyectos y grupos de estudio dentro y fuera de la institución escolar e intenta buscar, en el otro y con el otro, nuevas experiencias y saberes de la profesión docente ${ }^{85}$.

Esta visión del aprendizaje desde un enfoque humanístico privilegia el aprendizaje del profesor desde perspectivas socioculturales. En esta visión se encuentran diversas formas de asumir los contextos de desarrollo del profesor bajo la denominación de procesos de socialización del profesor de matemáticas. Las diferentes visiones del problema del contexto resaltan en algunos casos que la actividad social es el propio contexto, y en otros que el contexto se constituye entre personas comprometidas en una actividad socialmente construida y el mundo en el que se mueven.

Estas interpretaciones y relaciones establecidas a través de los desarrollos investigativos reconocen prioritariamente el aprendizaje del profesor desde la perspectiva cognitiva. El énfasis en esta perspectiva ha generado que las investigaciones,

[...] se sitúen sobre el contenido y los procesos de pensamiento de los profesores; cómo el conocimiento está organizado y cómo se usa en contextos específicos (...). De ahí que el conocimiento y las creencias - algunas veces con la denominación de concepciones -, como estructuras mentales, sean los protagonistas de la mayoría de

82 Bárbara Jaworski, “Theory and practice in mathematics teaching development:critical inquiry as a mode of learning teaching”, en Journal of Mathematical Teacher Education 9 (2006): 189.

83 Darío Fiorentin y M.A Miorim, M.A, "Por trás da porta, que Matemática acontece? (Campinas: Editora Gráfica FE/Unicamp, 2001a). Darío Fiorentini y M. A Miorim, "Pesquisar \& escreve também é preciso: a trajetória de um grupo de professores de matemática, En Por trás da porta, que Matemática acontece? Org. Dario Fiorentini y M. A Miorim, (Campinas: Editora Gráfica FE/Unicamp, 2001b): 12-30.

Darío Fiorentini, "Learning and professional development of mathematics teacher in research communities", Sisyphus-Journal of Education 1, No. (3) (2013): 152-181.

Renata Gama y Darío Fiorentini, "Formação continuada em grupos colaborativos: professores de matemática iniciantes e as aprendizagens da prática profissional" Educación Matematica Pesq 11, No. 2(2009): 441-461.

Emerson Gómez, "Aprendizagem docente e desenvolvimento profissional de professores de matemática investigação de experiências colaborativas no contexto da amazônia paraense (Tesis de Doctorado en, Brasil: Universidade Federal de Mato Grosso, 2014).

84 Fiorentini, "De professor isolado ou plugado para professor conectado: novas perspectivas à formação do professor de matemática".

85 Alfonso Jiménez Espinosa, "Quando professores da Matemática da escola e da universidade se encontram:re-signifição e reciprocidade de saberes”, (Tese de Doutorado em Educação; Universidade Estadual de Campinas, 2002.

Alfonso Jiménez Espinosa, Formación de profesores de matemáticas: aprendizajes recíprocos escuela-universidad. (Tunja: Búhos Editores, 2005). 
los estudios realizados con una atención sobre la forma en que se conoce o cree, cómo están organizados, cómo cambian o cómo determinan el aprendizaje y la práctica ${ }^{86}$.

De otro lado, recientemente se ha abordado el desarrollo investigativo desde las perspectivas socioculturales, lo cual ha atribuido nuevos significados tanto al conocimiento como al proceso de conocer.

La emergencia de estas perspectivas sitúa el conocer-como un atributo tanto del individuo que participa en una comunidad de aprendices en el proceso de aprender a enseñar, o de profesores de matemáticas considerando la práctica de enseñar matemáticas-como un atributo de las propias comunidades a las que se pertenece ${ }^{87}$.

\section{Dilemas sobre el aprendizaje del profesor de Matemáticas}

Las diferentes visiones sobre el aprendizaje del profesor de matemáticas presentanherramientasconceptualesdegraninterés, propicias para problematizar el proceso de aprendizaje del profesor y comprender que los dilemas se enmarcan en los presupuestos que subyacen al interior de posicionamientos teóricos establecidos. Las perspectivas generales sobre el aprendizaje sugieren que el punto central de la problematización es un asunto epistemológico ${ }^{88}$, ya que, por ejemplo, algunos lo consideran como un proceso de internalización de entidades reales existentes en la mente del que aprende, o quienes adoptan una visión del conocimiento y el aprendizaje como la participación en cambiantes procesos de actividad humana ${ }^{89}$, solo por mencionar dos posturas.

Este asunto epistemológico destaca limitaciones que varían desde entender el aprendizaje como un proceso contenido en la mente del que aprende, que ignora el mundo en que se vive y que se concentra estrictamente en la trasmisión del conocimiento existente; que además guarda silencio respecto a la invención de nuevo conocimiento en la práctica, hasta asumir una supuesta homogeneidad de actores, objetivos, motivos y actividades. Estas limitaciones plantean dilemas conceptuales como la dicotomía del cuerpo y la mente; investigar la práctica concentrada en las actividades de las personas que actúan, y dejar de lado el mundo social en que la actividad se desarrolla.

Estos dilemas constituyen lo que Wenger ${ }^{90}$ ha denominado "problema de contexto", al referirse a la forma como las teorías convencionales del aprendizaje y la enseñanza apelan al carácter descontextualizado de ciertos conocimientos

\footnotetext{
86 Llinares, "La investigación <sobre> el profesor de matemáticas: aprendizaje del profesor y práctica profesional", p. 178).

87 Gómez, "Teorías de aprendizaje y formación de profesores," 178.

88 Wenger, Comunidades de Práctica. Aprendizaje, significado e identidad

89 Jean Lave, "Situating learning in communities of practice," en Perspective on socially shared cognition, editado por Lauren Resnick, Levine B., M. John, Stephanie Teasley (Washington: American Psycological association, 1991): 63-82.

90 Wenger, Comunidades de Práctica. Aprendizaje, significado e identidad
} 
y atribuir posibles formas de trasmisión de los mismos. En Lave ${ }^{91}$ el contexto se considera:

[...] como un mundo social constituido en relación con personas actuantes, tanto el contexto como la actividad parecen inexorablemente flexibles y cambiantes. Desde esta perspectiva, la participación y la comprensión cambiantes en la práctica -el problema de aprendizaje- no pueden sino tornarse también centrales.

Este concepto de contexto se presenta con la intención de ampliar la visión del aprendizaje desde las propuestas cognitivistas hasta las diferentes propuestas de la actividad situada. Las primeras plantean una dicotomía del cuerpo y la mente, pues asumen el aprendizaje como un proceso contenido en la mente del que aprende e ignoran el mundo en el que vive; las segundas no establecen una separación entre acción, pensamiento, sentimiento y valor y sus formas colectivas e histórico-culturales de actividad localizada, interesada, conflictiva y significativa. Sin embargo, estas últimas enmarcadas dentro de una perspectiva sociocultural difieren en algunos presupuestos, como también se soportan en otros puntos de acuerdo.

Dentro de los acuerdos se resalta que el aprender no es problemático, pero lo aprendido sí lo es y que la mediación histórica en este proceso es fundamental. Las discrepancias se relacionan con la forma de abordar la mediación histórica. Las teorías fenomenológicas sostienen que la actividad social es el propio contexto, luego la relación teórica central es la relación intersubjetiva entre quienes coparticipan en la interacción social; se fundamenta en la premisa de que la situaciones se construyen, mientras las personas se organizan para atender y dar significado a sus preocupaciones ${ }^{92}$. En tanto las teorías de la actividad situada cuestionan estos supuestos sobre la base de puntualizar que la conexión y el significado de la actividad no puede explicarse desde el análisis de las situaciones inmediatas de las acciones específicas o de las personalidades de los participantes; plantea que la relación teórica central se constituye entre personas comprometidas en una actividad socialmente construida y el mundo en el que se mueven ${ }^{93}$; de esta forma el significado no se crea por intenciones individuales, sino que se constituye mutuamente en las relaciones entre sistemas de actividad y personas que actúan y tienen un carácter relacional.

Estas diversas formas de precisar conceptualmente el contexto dan la posibilidad de entender la acción y sus relaciones, las cuales, en este caso, proveerían de significado al aprendizaje. El aprendizaje como participación se encuentra en el medio de estos dos puntos de vista, enmarcado dentro de las teorías de la estructura social y las teorías de la experiencia situada; es decir, "tiene lugar mediante nuestro compromiso en acciones y en interacciones, pero enmarca este compromiso en la cultura y en la historia. Mediante estas acciones

91 Lave, "Situating learning in communities of practice", 17.

92 Lave, "Situating learning in communities of practice"

93 Lave, "Situating learning in communities of practice" 
e interacciones locales, el aprendizaje se produce y transforma la estructura social en la que tiene lugar"94. La postura de Lave, en concordancia con la forma en que Wenger presenta el aprendizaje, replantea el problema del contexto, en la medida en que reconoce que las diversas formas de abordarlo, desde el punto de vista social están focalizadas en determinar la relación constitutiva entre las personas que actúan y los contextos con los cuales actúan; a cambio Lave propone cuestionar "[...] cuáles son las relaciones entre las prácticas locales que contextualizan las maneras en que las personas actúan juntas, tanto en los contextos como a través de ellos" ${ }^{\prime \prime 5}$.

Este problema de contexto permite establecer una marcada diferencia entre teorías del aprendizaje inscritas en la metáfora de la adquisición y la metáfora del aprendizaje como participación en una comunidad de prácticas basada en la cognición situada ${ }^{96}$.

\section{CONCLUSIÓN}

Con la revisión realizada se puede concluir que el aprendizaje del profesor, por mucho tiempo y a través del análisis de muchas investigaciones se interpreta prioritariamente desde la perspectiva cognitiva, excepto algunos casos derivados de interpretaciones desde las perspectivas socioculturales o antropológicas. El énfasis en la perspectiva cognitiva ha generado que las investigaciones:

[...] se sitúen sobre el contenido y los procesos de pensamiento de los profesores, cómo el conocimiento está organizado y cómo se usa en contextos específicos. La perspectiva cognitiva asume que las estructuras de conocimiento del individuo y las representaciones mentales del mundo son variables en el aprendizaje y en la práctica. De ahí que el conocimiento y las creencias -algunas veces con la denominación de concepciones-, como estructuras mentales, sean los protagonistas de la mayoría de los estudios realizados con una atención sobre la forma en que se conoce o cree, cómo están organizados, cómo cambian o cómo determinan el aprendizaje y la práctica97.

De otro lado, las perspectivas socioculturales han atribuido nuevos significados tanto al conocimiento como al proceso de conocer. Por ejemplo:

[...] la emergencia de las perspectivas situadas sobre la actividad y el aprendizaje ven el conocimiento como distribuido entre las personas y sus entornos, lo que incluye tanto los artefactos de la práctica como las comunidades en las que se toma parte. Desde estas perspectivas el análisis de la actividad se centra entre las interacciones

94 Wenger, Comunidades de Práctica. Aprendizaje, significado e identidad, 31.

95 Lave, "Situating learning in communities of practice," 34.

96 Anna Sfard, “On Two Metaphors for Learning and the Dangers of Choosing Just One," en Educational Research 27, no 2 (1998): 4-13.

97 Llinares, "La investigación <sobre> el profesor de matemáticas: aprendizaje del profesor y práctica profesional”, 178. 
entre los individuos y entre estos y los sistemas físicos y tecnológicos en los que se está. La emergencia de estas perspectivas sitúa el «conocer» como un atributo tanto del individuo que participa en una comunidad [de aprendices en el proceso de aprender a enseñar, o de profesores de matemáticas considerando la práctica de enseñar matemáticas], como un atributo de las propias comunidades a las que se pertenece ${ }^{98}$.

Esta visión de aprendizaje permite establecer marcadas diferencias con otras visiones, y deja así abiertas posibilidades de análisis del aprendizaje del profesor desde perspectivas complementarias o perspectivas de profundización desde el enfoque sociocultural que vienen resaltando la necesidad de abrir agendas de investigación sobre ¿cómo los profesores aprenden ${ }^{99}{ }^{9}$ y cómo ellos dan sentido a sus situaciones de enseñanza y aprendizaje de las matemáticas ${ }^{100}$. Así como también resaltan la necesidad de vincular el aprendizaje con el desarrollo profesional $^{101}$.

Desde una perspectiva sociocultural, particularmente la visión de aprendizaje del profesor como participación, no la reduce al análisis solo de los mecanismos cognitivos del individuo, que se adquieren o se construyen suponiendo la existencia de unos entes mentales que permiten explicar e interpretar las actuaciones de los sujetos; sino que deja abierta posibilidades de análisis del aprendizaje a partir de constructos conceptuales como significado, identidad, práctica y comunidad.

En consecuencia, el aprendizaje del profesor de matemáticas es un proceso complejo que exige la conceptualización, elaboración de modelos y la teorización como tres etapas de desarrollo ${ }^{102}$. De esta forma, la adopción de nuevos posicionamientos epistemológicos en campos específicos de investigación sobre el análisis de la práctica del profesor propone nuevas problemáticas de investigación.

\section{REFERENCIAS}

98 Llinares, "La investigación <sobre> el profesor de matemáticas: aprendizaje del profesor y práctica profesional”, 178.

99 Llinares, "Experimentos de enseñanza e investigación"

100 Salvador Llinares, “¿Cómo dar sentido a las situaciones de enseñanza- aprendizaje de las Matemáticas? Algunos aspectos de la competencia docente del profesor", Cuadernos de Investigación y formación en Educación Matemática 15, (2016): 57-67.

G. Sánchez-Matamoros, C. Fernández, y Salvador Llinares, "Developing pre- service teachers' noticing of students' understanding of the derivative concept", International Journal of Science and Mathematics Education, 13, (2015): 1305- 1329. DOI: 10.1007/s10763014-9544-y

101 Fiorentin y Miorim, "Por trás da porta, que Matemática acontece?

Fiorentini y Miorim, "Pesquisar \& escreve também é preciso: a trajetória de um grupo de professores de matemática"

Fiorentini, "Learning and professional development of mathematics teacher in research communities

Gama y Fiorentini, "Formação continuada em grupos colaborativos: professores de matemática iniciantes e as aprendizagens da prática profissional"

Gómez, "Aprendizagem docente e desenvolvimento profissional de professores de matemática investigação de experiências colaborativas no contexto da amazônia paraense"

102 Lerman, "A rewiew of research perspectives on mathematics teacher education" 
Adler, Jill y Barbara Jaworski. "The state of research on mathematics teacher education, and how it needs to develop," Trabajo presentado en The Fifteenth ICMI Study. Aguas de Lindóia, 2005.

Adler, Jill, Deborah Ball, Konrad Krainer, Fou-Lain Lin, y Jarmila Novotna, "Mirror images of an emerging field: researching teacher education " en Proceedings from the ICME 10. Dinamarca: IMFUFA, Departament of Sicence, Systems and Models, Roskilde University Denmark, 2008, 123-139.

Alsina, Ángel. “El aprendizaje reflexivo en la formación inicial del profesorado: un modelo para aprender a enseñar matemáticas", Educación Matemática.Vol. 22 No. 1 (2010):149.

Azcárate, Pilar. "La formación inicial del profesor de matemáticas: análisis desde la perspectiva del conocimiento práctico profesional", Revista Interuniversitaria de Formación del Profesorado (1998): 129-142.

Ball, Débora, Mark Thames y Geoffrey Phelps, “Content knowledge for teaching: What makes it special?", Journal of Teacher Education 59, No 5 (2008): 389-407.

Blanco, Lorenzo. "Problem solving and the initial and theoretical education of teachers in Spain," en Mathematics Teacher Educational and Development 6, (2004):37-48.

Brown, Catherine y Hilda Borko, "Becoming a Mathematics Teachers", en Handbook of Research on Mathematics Teaching and Learning, editado por A. Grouws. New York: MacMillan,1992, 209-239.

Brown, Stephen, Thomas Cooney, Douglas Jones. "Mathematics Teacher Education" en Handbook of Research on Teachers Education, editado por W. Houston. New York: MacMillan, 1990.

Camargo, Leonor. “Descripción y análisis de un caso de enseñanza y aprendizaje de la demostración en una comunidad de práctica de futuros profesores de matemáticas de educación secundaria". Tesis de Doctorado en Universidad de Valencia, Valencia, España: 2010.

Chávez, Y. y Salvador Llinares, "La identidad como producto del aprendizaje en la práctica de enseñar matemáticas en profesores de primaria”. En Investigación en Educación Matemática XVI, editado por A. Estepa, A. Contreras, J. Deulofeu, M. Penalva, F., y L. Ordóñez. Jaén: SEIEM,2012, 187-196.

Cooney, Thomas. "Research and Teacher Education: in search of common ground", en Journal for Research in Mathematics Educations 25, (1994): 608-636.

Cooney, Thomas y T. Wilson. "Teachers Thinking About Functions: Historical and Research Perspectives", en Integrating Research on the Graphical Representation of Functions, editado por T. Romberg, E. Fennema, y T. Carpenter. Hillsdale, NJ: Lawrence Erlbaum Associates, 1994.

Corral, C y E. Zurbano. Propuestas metodológicas y de evaluación en la formación inicial de los profesores del área de didáctica de la matemática. Oviedo: Servicio de Publicaciones Universidad de Oviedo, 2000. 
Feiman-Nemser, Sharon. "Teachers preparation:structural and conceptual alternatives" en Handbook of research on teacher education, editado por W. Houston. New York: MacMillan,1990.

Ernest, Paul. "What is social constructivism in the psychology of mathematics education?" en Prodeedings of the Eighteenyh International Conference for PME, editado por Joao Ponte, y Joao Filipe Matos. Lisboa , 1994, 304-311.

Fenstermacher, Gary. "The Knower and the Know: The Nature of Knowledge in Research in Education", en Review of Research in Education, editado por L. DarlingHammond. Washington: AREA, 1994.

Fiorentini, Darío. “De professor isolado ou plugado para professor conectado: novas perspectivas à formação do professor de matemáticas," en Coletânea de trabalhos do PRAPEM-VII ENEM. Campinas: CEMPEM/PRAPEM-FE/UNICAMP, 2001.

Fiorentin, Darío y M.A Miorim, M.A, "Por trás da porta, que Matemática acontece?. Campinas: Editora Gráfica FE/Unicamp, 2001a.

Fiorentini, Darío y M. A Miorim, “Pesquisar \& escreve também é preciso: a trajetória de um grupo de professores de matemática, en Por trás da porta, que Matemática acontece?, org. Dario Fiorentini y M. A Miorim. Campinas: Editora Gráfica FE/ Unicamp, 2001b, 12-30.

Fiorentini, Dario. "Learning and professional development of mathematics teacher in research communities", Sisyphus-Journal of Education 1, No.(3) (2013): 152-181.

Fiorentini, Darío y Ana Teresa de Carvalho Correa de Oliveira, "Lugar das matemáticas na Licenciatura em Matemáticas: que matemáticas e que práticas formativas" Boletim de Educação Matemática 27, No 47 (2013): 917-938.

Flores, Pablo. “Concepciones y creencias de los futuros profesores sobre las matemáticas, su enseñanza y aprendizaje". Tesis de Doctorado en Universidad de Granada, 1998.

Gama, Renata y Darío Fiorentini, "Formação continuada em grupos colaborativos: professores de matemática iniciantes e as aprendizagens da prática profissional" Educación Matematica Pesq 11,no 2(2009): 441-461.

García Blanco, Maria Mercedes. “La formación inicial de profesores de matemáticas. Fundamentos para la definición de un currículum", en A formação de professores de matemática.Estudos e contribuições teorico-metodologicas de Brasil Espanha e Portugal, editado por Dario Fiorentini. Campinas, Brasil : UNICAMP, 2001

García Blanco, Maria Mercedes. “La Formación deProfesores de Matemáticas: un campo de estudio y preocupación”. Educación Matemática 17, No. 2 (2005): 153-166.

Giménez, Joaquín, Salvador Llinares y Victoria Sánchez, El proceso de llegar a ser un profesor de matemáticas. Cuestiones desde la Educación Matemática. Granada: Comares, 1996.

Gómez, Emerson. “Aprendizagem docente e desenvolvimento profissional de professores de matemática investigação de experiências colaborativas no contexto da amazônia paraense. Tesis de Doctorado en Brasil: Universidade Federal de Mato Grosso, 2014. 
Gómez, Pedro. “Teorías de aprendizaje y formación de profesores”, en El practicum en la formación del profesorado de magisterio y educación secundaria: avances de investigación, fundamentos y programas de formación . Granada: Universidad de Granada, 2002, 459-467.

Gómez, Pedro. "Procesos de Aprendizaje en la Formación Inicial de Profesores de Matemáticas de Secundaria", Electronic Journal of research in Educational Psychology 7, No 1 (2009): 471-498.

Hersant, M. y Marie-Jeanne Perrin-Glorian. "Characterization of an Ordinary Teaching Practice with the Help of the Theory of Didactic Situations", Educational Studies in Mathematics, 59, No 1-3 (2005): 113-151.

Jaworski, Barbara. "Theory and practice in mathematics teaching development:critical inquiry as a mode of learning teaching", en Journal of Mathematical Teacher Education 9 (2006): 189.

Jaworski, Bárbara. "Building and sustaining inquiry communities in mathematics teaching development. Teachers and didacticians in collaboration", en Participants in Mathematics Teachers Education, editado por K. Krainer, y T. Wood. Sence Publishers. All rights reserved, 2008, 309-33.

Jiménez Espinosa, Alfonso. "Quando professores da Matemática da escola e da universidade se encontram:re-signifiçao e reciprocidade de saberes. Tesis de Doctorado en Universidad Estadual de Campinas, 2002.

Jiménez Espinosa, Alfonso. Formación de profesores de matemáticas: aprendizajes recíprocos escuela-universidad. Tunja: Búhos Editores, 2005.

Krainer, Konrad. "Individuals, teams communities and networks: participants and ways of participation in mathematics teacher education," en Participants in Mathematics Teacher Education, editado por.Konrad Krainer y Terry Wood. Rotterdam, Sence Publishers, 2008, 10.

Lave, Jean . "Situating learning in communities of practice," en Perspective on socially shared cognition, editado por Lauren Resnick, Levine B., M. John, Stephanie Teasley. Washington: American Psycological association, 1991, 63-82.

Lerman, Stephen. "Metaphors for mind aand mataphors for teaching and learning mathematics," en Proceeding of the Eigghteenth International Conference for PME, III, J editado por Joao Ponte, y Joao Filipe Matos. Lisboa, 1994, 144-151.

Lerman, Stephen. "A rewiew of research perspectives on mathematics teacher education", en Making sence of mathematics teacher education. Dordrecht, The Netherlands: Kluwer Academic Publishers, 2001, 33-52.

Lerman, Stephen. "Socio-cultural research in PME", en Handbook of Research on the Psichology of Mathematics: past, Present and Future. Rotterdam-Tapei: Sence Publishers, 2006, 347-366.

Lerman, Stephen y Stefan Zehetmeier, "Face-to-Face Communities and Networks of Practicing Mathematics Teachers Studies on their professional growth,"en 
Handbook Communities and Networks of Mathematics Teachers as Learners 3, editado por Konrad Krainer y Terry Wood. Rotterdam: Sence Publishers, 2008, 133-154.

Lin, Fou-Lain y Thomas Cooney. Making sence of mathematics teacher education (Dordrecht The Netherlands: Kluwer, 2001) citado por Cristina Esteley, "Desarrollo Profesional en Escenarios de Modelación Matemática: voces y sentidos" (Tesis de Doctorado en Universidad Nacional de Cordoba, 2010.

Llinares, Salvador. "La investigación <sobre> el profesor de matemáticas: aprendizaje del profesor y práctica profesional”. Aula 10, (1998):153-179.

Llinares, Salvador. "Relación entre teorías sobre el aprendizaje del profesor de matemáticas y diseños de entornos de aprendizaje," en Conferencia invitada presentada en el Congreso Iberoamericano de Educación Matemática- CIBEM. Portugal, 2005.

Llinares, Salvador. “Formación de Profesores de Matemáticas. Desarrollando Entornos de Aprendizaje ára relacionar la formación inicial y el desarrollo profesional. Conferencia invitada en el XIII Jornadas de Aprendizaje y Enseñanza de las Matemáticas. Granada, España, julio de 2007.

Llinares, Salvador. "Aprendizaje del estudiante para profesor de matemáticas y el papel de los nuevos instrumentos de comunicación," en conferencia realizada en el marco del III Encuentro de Programas de Formación Inicial de Profesores, Universidad Pedagógica Nacional en Colombia, 2008 http://rua.ua.es/dspace/ bitstream/10045/5302/1/llinares-bogota08.pdf

Llinares, Salvador. “Experimentos de enseñanza e investigación. Una dualidad en la práctica del formador de profesores de matemáticas", Educación Matemática, $n^{\underline{o}}$ extraordinario XXV aniversario, (2014): marzo, 31-51.

Llinares, Salvador. “¿Cómo dar sentido a las situaciones de enseñanza- aprendizaje de las Matemáticas? Algunos aspectos de la competencia docente del profesor", Cuadernos de Investigación y formación en Educación Matemática 15, (2016): 57-67.

Margolinas, C. y M. J. Perrin-Glorian.“Des recherches visant à modéliser le rôle de l'enseignant", Recherches en didactique des mathématiques 17, No. 3 (1997): 7-15.

Melo, J. “ A formação do formador de professores de matemática no contexto das mudanças curriculares. Tesis de doctorado en, Campinas, São Paulo, Brasil: Universidade Estadual de Campinas, 2010.

Oliveira, Hélia y Markku Hannula. "Individual prospective mathematics teachers," en The International Handbook of Mathematics Teachers Education 3, editado por Konrad Krainer y Terry Wood. Rotterdam: Sence Publishers, 2008, 13-34.

Peñas, María. “Un estudio del proceso de reflexión sobre cuestiones profesionales en la formación inicial de profesores de matemáticas," en Sexto Simposio de la Sociedad Española de Investigación en Educación Matemática, editado por Jesús Murillo, Petra María Arnal, Rafael Escolano, José María Gairín, y Lorenzo Jesús Blanco. SEIEM, Universidad de la Rioja, Departamento de Matemáticas y Computación, 2003. 
Perrin-Glorian, Marie-Jeanne, Lucie DeBlois y Aline Robert. "Participants in Mathematics Teacher Education: Individual, Teams, Communities and Networks," en The International Handbook of Mathematics Teacher Education 3, editado por Konrad Krainer y Terry Wood. Rotterdam: Sence Publishers, 2008, 35-59.

Prescott y M. Cavanagh, "A situated Perspective on Learning to Teach Sencondary Mathematics", En Proceedings of the 31st Annual Conference of the Mathematics Education Research Group of Australian, editado por R. Goos, R. Brown y K. Makar. Merga Inc,2008.

Sánchez García, Victoria. “La formación de los profesores y las matemáticas. Algunas implicaciones prácticas de las investigaciones". Revista de Educación, No. 306 (1995): 397-426.

Sánchez-matamoros,G., C. Fernández, y Salvador Llinares. “Developing preservice teachers' noticing of students' understanding of the derivative concept", International Journal of Science and Mathematics Education, 13, (2015): 1305- 1329. DOI: 10.1007/s10763-014-9544-y

Schön, Donald. The reflective practitioner. New York: Basic Books, 1983.

Schön, Donald. Educating the reflective practitioner.Toward a new desig for teaching and learning in the professions. San Francisco: Jossey- Bass, 1987.

Sfard, Anna. "On Two Metaphors for Learning and the Dangers of Choosing Just One," en Educational Research 27, No. 2 (1998): 4-13.

Shulman, Lee. "Those who understand: knowledge growth in teaching", en Educational Researcher, 1986, 4-14.

Shulman, Lee. "Renewing the pedagogy of teacher education: The impact of subjectspecific conceptions of teaching", en Las didácticas específicas en la formación de profesores, editado por L. Moreno y J.M. Vez. Santiago: Tórculo, 1993, 53-69.

Shulman, Lee. "Foreword", en Examining Pedagogical Content Knowledge, editado por J. Gess-Newsome y N. Lederman. Londres: Kluwer Academic Publishers, 1999, ix-xii.

Vergnaud, Gérard. "Epistemology and psychology of mathematics education" en Research Sinthesis by International Group for the Psychology of Mathematics Education, editado por J. Pearla Nesher y Jeremy. Kilpatrick. New York: Cambridge University Press, 1990, 14-30.

Vigostki, Lev. Pensamiento y Lenguaje. La Habana: Editorial Revolucionaria, 1978.

Von Glasersfeld, Ernest. “Cognition, construction of knowledge, and teaching”, Synthese 80, (1989):121-140.

Wenger, Etiene. Comunidades de Práctica. Aprendizaje, significado e identidad (Barcelona: Ediciones Paidós Ibérica, S. A, 2001).

Wenger, Etiene . "Uma teoria social da aprendizagem”, en Teorias contemporâneas da aprendizagem, editado por Knud Illeris. Porto Alegre: Penso, 2013. 
Yáñez, José Carrillo y Nuria de los Ángeles Climent Rodríguez, Modelos de formación de maestros en matemáticas. Huelva: Servicio de Publicaciones de la Universidad de Huelva,1999.

Zeichner, Kenneth y J. Gore. "Teacher Socialization”, Paper presented at the annual meeting of the Association of Teacher Educators. Las Vegas, February de 1990.

Zeichner, Kenneth. "Connecting genuine teacher development to the struggle for social justice" en Journal of Education for Teaching 19, No 1 (1993): 5-20.

Riscanevo Espitia, Lida Esperanza: Jiménez Espinosa, Alfonso. "El aprendizaje del profesor de matemáticas como campo investigativo". Revista Historia de la Educación Latinoamericana. Vol. 19 No. 28 (2017): 173-196 